\title{
Association between Childhood Obesity and Neighbourhood Accessibility to Fast-Food Outlets: A Nationwide 6-Year Follow-Up Study of 944,487 Children
}

\author{
Tsuyoshi Hamano ${ }^{\text {a, b }}$ Xinjun Lic Jan Sundquist ${ }^{c, d}$ Kristina Sundquist ${ }^{c, d}$ \\ ${ }^{a}$ Department of Sports Sociology and Health Sciences, Faculty of Sociology, Kyoto Sangyo \\ University, Kyoto, Japan; ${ }^{b}$ Department of Functional Pathology, Shimane University School of \\ Medicine, Izumo, Japan; 'Centre for Primary Health Care Research, Lund University, Malmö, \\ Sweden; ${ }^{\mathrm{d}}$ Department of Family Medicine and Community Health, Department of Population \\ Health Science and Policy, Icahn School of Medicine at Mount Sinai, New York, NY, USA
}

\section{Keywords}

Childhood obesity · Fast-food outlets · Follow-up study · Multilevel analysis

\begin{abstract}
Objectives: The aim of this 6-year follow-up study was to examine whether neighbourhood accessibility to fast-food outlets was associated with diagnosed childhood obesity, after adjustment for neighbourhood- and individual-level socio-demographic factors. Methods: This 6-year follow-up study comprised 484,677 boys and 459,810 girls aged 0-14 years in Sweden. The follow-up period ran from January 1, 2005, until hospitalisation/out-patient treatment for obesity, death, emigration or the end of the study period on December 31, 2010. Multilevel logistic regression models (individual-level factors at the first level and neighbourhood-level factors at the second level) were used to calculate odds ratios (ORs) with $95 \%$ confidence intervals (95\% CIs). Results: We identified 6,968 obesity cases (3,878 boys and 3,090 girls) during the follow-up period. Higher odds of childhood obesity for those living in neighbourhoods with accessibility to fast-food outlets was observed $(\mathrm{OR}=1.14,95 \% \mathrm{CI}=1.07-1.22)$ that remained significant after adjustments $(O R=1.06,95 \% C I=1.00-1.13)$. Conclusions: This prospective nationwide study showed that the neighbourhood accessibility to fast-food outlets was independently associated with increased odds of diagnosed childhood obesity. This finding implicates that residential environments should be considered when developing health promotion programmes.

(C) 2017 The Author(s)

Published by S. Karger GmbH, Freiburg
\end{abstract}




\section{Introduction}

Childhood obesity is one of the major public health concerns, and its prevalence has increased over the years [1]. Previous studies found that risk factors for childhood obesity include genetic [2] and lifestyle factors (e.g. lack of physical activity and excess and unhealthy food intake) [1]. Neighbourhood environments have been also considered to be an important risk factor for childhood health problems including obesity [3-5]. Recent research on neighbourhood environments and health has been driven by two trends within public health and epidemiology [3]:

The first trend is a focus on the neighbourhood social environment that is measured using census characteristics such as socio-economic composition and crime statistics [3]. For example, a previous follow-up study conducted in Sweden found that neighbourhood deprivation was independently associated with increased odds of diagnosed childhood obesity [5]. In addition, a systematic review in the UK found that higher neighbourhood-level deprivation was positively associated with childhood obesity prevalence [6].

The second trend is a focus on the neighbourhood physical environment that is measured by access to food outlets (e.g. population size per fast-food restraint, distance to usual grocery and fast-food restaurant proximity), access to physical activity (e.g. proximity to play space/ recreational facilities, walkability and sidewalk availability) and other measures (e.g. daily amount of time spent in a car, amount of greenery and metropolitan sprawl index) [3, 7].

Fast-food diets are often convenient and readily accessible. However, a previous study suggested that children who ate fast food, compared with those who did not, consumed more total energy [8]. Therefore, an important hypothesis to examine is that neighbourhood accessibility to fast-food outlets could be a predictor of childhood obesity. A number of studies have examined this hypothesis, with mixed findings. For example, a previous cross-sectional study conducted in the UK found that accessibility to fast-food outlets was associated with childhood obesity [9]. Conversely, a 3-year follow-up study conducted in the US could not find similar associations [10]. In such studies, longer follow-up periods and larger samples are needed to further disentangle the possible association between neighbourhood accessibility to fastfood outlets and childhood obesity.

It is also important to note that multilevel modelling is needed when examining the possible association between neighbourhood accessibility to fast-food outlets and childhood obesity, since this association could be confounded by neighbourhood- and individual-level socio-demographic factors [7]. However, few studies have investigated whether accessibility to fast-food outlets is associated with diagnosed childhood obesity, after adjustment for neighbourhood deprivation and individual-level covariates by using a multilevel statistical model on a large sample and with an adequate follow-up.

The aim of this 6-year follow-up study in the entire Swedish population was to examine whether neighbourhood accessibility to fast-food outlets is associated with diagnosed childhood obesity. The second aim was to test whether these possible associations remain significant after adjustment for neighbourhood- and individual-level socio-demographic factors.

\section{Material and Methods}

Data used in this study were retrieved from national registers that contains information on the entire population of Sweden. The dataset we used contains nationwide information on parents and their offspring at the individual and neighbourhood level, including comprehensive demographic and socio-economic data. The information used in the present study comes from several Swedish national registers. The registers used in the present study were the Total Population Register, the Multi-Generation Register, the Hospital Discharge Register and the Outpatient Register. The Swedish nationwide population and health care registers have exceptionally high completeness and validity [11]. Individuals (children and their parents) were tracked 
using the personal identification numbers, which are assigned to each resident of Sweden. These identification numbers were replaced with serial numbers to provide anonymity. The follow-up period ran from January 1, 2005 until hospitalisation/out-patient treatment for obesity, death, emigration or the end of the study period on December 31, 2010.

\section{Outcome Variable: Diagnosed Childhood Obesity}

The outcome variable in this study was a hospital or out-patient diagnosis of childhood obesity (age at diagnosis 0-14 years) during the study period. Data on in-patient and out-patient diagnoses of obesity for 2005-2010 were retrieved from the Hospital Discharge Register and Out-Patient Register, which contain information on all hospital visits, including diagnoses. We searched these two registers for the International Classification of Diseases 10 (ICD-10) codes E65 and E66, denoting obesity as the main diagnosis during the study period. The serial numbers were used to ensure that each individual appeared only once in the dataset for his or her first diagnosis of obesity during the study period. Males and females with pre-existing obesity, defined as hospitalisation $\leq 5$ years before the start of the study, were excluded.

\section{Predictor Variable: Accessibility to Fast-Food Outlets}

This study used ready-to-use nationwide Geographic Information Systems (GIS) datasets of business contacts (i.e. goods, services and resources) that was provided to us by the Swedish company Teleadress [12], the leading provider of Swedish contact information, to collect data on fast-food outlets. Teleadress provides information on practically all businesses and services in Sweden with a registered telephone number and/or businesses that have provided information about their existence to the company. Both government and private entities are included. The database is updated continuously and has a high level of completeness of the data [13]. Data was drawn from 2005, i.e. at baseline.

Neighbourhood accessibility to fast-food outlets (e.g. pizzerias and hamburger outlets) was measured in 2005 as counts per pre-defined area called Small Area Market Statistics (SAMS, provided by Statistics Sweden) by using GIS. Accessibility was defined as the presence within the SAMS unit of at least one feature for the fast-food outlets. The presence of yes/no was chosen to define neighbourhood accessibility rather than linear density because the large majority had no access to the studied resources [13].

In the present study, neighbourhood of residence was provided to us by the Statistics Sweden via data from the National Land Survey. The home addresses of all individuals had been previously geocoded, allowing us to identify the SAMS units where the participants lived. However, the researchers in the present study had no access to the home addresses of the individuals, in order to ensure all individuals' integrity. SAMS units were used as proxies for neighbourhoods as have been used previously [14-17]. Each unit contains an average of approximately 1,000 residents in the whole of Sweden and around 2,000 in Stockholm. This study examined only those SAMS units that overlap with 'localities' or urban areas. In Sweden, 'localities' (which are defined by the Statistics Sweden every 5 th year) represent any village town, or city with a minimum of 200 residents and adjacent areas where the houses are no more than $200 \mathrm{~m}$ apart [18]. We chose to include only SAMS units overlapping with localities because more rural SAMS units have very few goods, services and resources. In 2005, 1,940 Swedish localities were identified by the Statistics Sweden. The GIS were used to overlay the SAMS boundaries with the locality boundaries. Of the total of 9,617 SAMS units in Sweden, 7,945 overlapped with localities and were therefore selected. SAMS units with fewer than 50 people were excluded on the basis that they might yield unreliable statistical estimates in the calculation of the neighbourhood deprivation index. Thus, a final total of 7,279 SAMS units were included in the present study.

In order to investigate the individuals' 'immediate' neighbourhood, we also used buffer zones. The Statistics Sweden provided information about the individuals' land coordinates in squares of $100 \times 100 \mathrm{~m}$, ensuring the integrity of the individuals. For each individual, a buffer zone with a radius of $1,000 \mathrm{~m}$ was applied, i.e. a distance that most people are willing to walk. Most previous studies used 0.5 mile $(\sim 800 \mathrm{~m})$ and/or 1 mile $(\sim 1,600 \mathrm{~m})$, which corresponds relatively well to the present study [19]. The number of fastfood outlets within the buffer zones was calculated using GIS. Accessibility was defined as the presence within the buffer zone of at least one feature for the fast-food outlets.

\section{Individual-Level Socio-Demographic Variables}

Sex of child: boy or girl.

Age ranged from 0 to 14 years and was divided into three categories: 0-4, 5-9 and 10-14 years.

Maternal marital status was categorised as i) married/cohabitating or ii) never married, widowed or divorced. 
Hamano et al.: Association between Childhood Obesity and Neighbourhood Accessibility to Fast-Food Outlets: A Nationwide 6-Year Follow-Up Study of 944,487 Children

Family income was calculated as annual family income divided by the number of people in the family. The family income measurement took into consideration the ages of the family members and used a weighted system whereby younger children were given lower weights than adolescents and adults. The final variable was calculated as empirical quartiles from the distribution.

Maternal and paternal educational levels were categorised as completion of compulsory school or less ( $\leq 9$ years), practical high school or some theoretical high school (10-11 years), and completion of theoretical high school and/or college ( $\geq 12$ years).

Maternal and paternal country of birth: These variables were categorised as Sweden, Western countries (Western Europe, the USA, Canada and Oceania) and others.

Maternal urban/rural status: These variables were included because access to preventive antenatal care may vary according to urban/rural status. Mothers were classified as living in a large city, a middle-sized town or a small town/rural area. Large cities were those with a population of $\geq 200,000$ (Stockholm, Gothenburg and Malmö); middle-sized towns were towns with a population of $\geq 90,000$ but $<200,000$; small towns were towns with a population of $\geq 27,000$ and $<90,000$; and rural areas were areas with populations smaller than those of small towns. This classification yielded three equally sized groups.

Mobility: Children were classified as having 'not moved' or having 'moved' to another neighbourhood with the same or a different level of deprivation within 5 years.

Maternal age at childbirth was classified as $<30,30-39$, and $\geq 40$ years, and paternal age at childbirth was classified as $<30,30-39$ and $\geq 40$ years.

Maternal, paternal and individual hospitalisations were defined as the first diagnosis from the Swedish Hospital Register during the follow-up period of i) diabetes (ICD-10 E10-E14), ii) chronic obstructive pulmonary disease (ICD-10 J40-J47), and iii) alcohol-related liver disease (ICD-10 F10 and K70).

Because obesity is known to cluster in families, children were classified according to whether or not they had a family history (parents or siblings) of hospitalisation because of obesity.

\section{Neighbourhood-Level Deprivation}

A summary index was calculated to characterise neighbourhood-level deprivation. The neighbourhood index was based on information about female and male residents aged 20-64 years because this age group represents those who are among the most socio-economically active in the population (i.e. a population group that has a stronger impact on the socio-economic structure in the neighbourhood than children, younger women and men and retirees do). The neighbourhood index was based on four items: low educational level ( $<10$ years of formal education), low income (income from all sources, including interest and dividends, $<50 \%$ of the median individual income), unemployment (excluding full-time students, those completing military service and early retirees) and receipt of social welfare. The index of the year 2000 was used to categorise neighbourhood deprivation as low (more than 1 SD below the mean), moderate (within 1 SD of the mean), and high (more than 1 SD above the mean) [20].

\section{Statistical Analyses}

The cumulative rate of obesity was calculated for the total study population and for each subgroup after assessment of neighbourhood of residence for children. Multilevel (hierarchical) logistic regression models were used to estimate odds ratios (ORs) and 95\% confidence intervals (95\% CIs). The analyses were performed using MLwiN version 2.35 (University of Bristol, Bristol, UK).

Random intercept multilevel logistic regression models were used to allow for the clustering of individuals within neighbourhoods and to estimate the variance in obesity risk that is attributable to neighbourhood characteristics. This approach was used to estimate the intra-class correlation coefficient (ICC), the proportion of variance in the outcome attributable to differences between individuals in different neighbourhoods, in contrast to differences between individuals within the same neighbourhood [21]. The ICC was estimated by applying the latent variable method as exemplified by

$$
\text { ICC }=V_{n} /\left(V_{n}+\pi^{2} / 3\right)
$$

where $V_{n}$ is the variance between neighbourhoods and $\pi^{2} / 3$ is the variance between individuals.

The proportion of the second-level variance explained by different variables was calculated as

$$
\mathrm{V}_{\text {EXPLAINED }}=\left(\mathrm{V}_{0}-\mathrm{V}_{1}\right) / \mathrm{V}_{0} \times 100
$$

where $V_{0}$ is the age-adjusted variance in the initial model and $V_{1}$ is the second-level variance in different models. 
Interaction tests were performed to examine potential interactions between neighbourhood deprivation and accessibility to fast-food outlets.

Ethical Considerations

This study was approved by the Ethics Committee at Lund University.

\section{Results}

Table 1 shows the population characteristics and number of childhood obesity occurrences. In the total study population (944,487 children), 48.7\% were girls, and approximately 1 in 2 children (47.4\%) were aged 10-14 years. A total of 6,968 children were diagnosed with obesity during the 6-year follow-up period (January 1, 2005 to December 31, 2010). Cumulatively diagnosed childhood obesity rates increased from 0.4 per 100 in neighbourhoods with low deprivation to 1.1 per 100 in neighbourhoods with high deprivation.

Table 2 shows the associations between childhood obesity and accessibility to fast-food outlets in the neighbourhoods (SAMS units). A statistically significant higher odds of childhood obesity for those living in neighbourhoods with accessibility to fast-food-outlets was observed in the unadjusted model $(\mathrm{OR}=1.14,95 \% \mathrm{CI}=1.07-1.22)$ (Model 1$)$. The association remained significant, after adjustment for neighbourhood deprivation, individual age and sex (OR = 1.07, 95\% CI $=1.00-1.14$ ) (Model 2). The association also remained significant, after adjustment for neighbourhood-level deprivation, individual-level age, sex, maternal marital status, family income, parental educational level, parental immigrant status, region of residence, mobility and parental age at child birth $(\mathrm{OR}=1.06,95 \% \mathrm{CI}=1.00-1.13)$ (Model 3). Finally, the association remained significant, after full adjustments of the covariates (OR = $1.06,95 \% \mathrm{CI}=1.00-1.13$ ) (Model 4). In addition, the interaction tests were performed to examine potential interactions between neighbourhood deprivation and accessibility to fastfood outlets. The result showed no significant interactions (data not shown).

Table 3 shows the associations between the childhood obesity and the 'immediate' neighbourhood accessibility to fast-food outlets in the buffer zones (radius 1,000 m). A statistically significant higher odds of childhood obesity for those living in 'immediate' neighbourhoods with accessibility to fast-food outlets was observed in Model 1 (OR $=1.07,95 \% \mathrm{CI}=1.01-$ 1.14). However, no significant associations were found, after adjustment for the covariates (Models 2-4).

\section{Discussion}

The main finding of this 6-year follow-up study is that the neighbourhood accessibility to fast-food outlets exerts an independent effect on the odds of childhood obesity (table 2). Although the OR of the association is not strong $(\mathrm{OR}=1.06,95 \% \mathrm{CI}=1.00-1.13)$, it is important to note that our multilevel model was adjusted for neighbourhood-level deprivation and a comprehensive set of individual-level covariates (i.e. age, sex, maternal marital status, family income, parental educational level, parental immigrant status, region of residence, mobility, parental age at child birth, family history of obesity as well as individual and parental hospitalisations). In addition, the crude OR implies that children exposed to fast-food outlets have a 14\% actually increased OR of developing obesity. The crude OR is often important for decisions concerning distribution of health care resources, whereas the adjusted ORs are important to determine potential mechanisms. 
Hamano et al.: Association between Childhood Obesity and Neighbourhood

Accessibility to Fast-Food Outlets: A Nationwide 6-Year Follow-Up Study of 944,487

Children

Table 1. Distribution of population, number of childhood obesity events, and age-standardised cumulative rates of obesity (per 100) by neighbourhood-level deprivation

\begin{tabular}{|c|c|c|c|c|c|c|c|c|c|}
\hline & \multicolumn{2}{|c|}{ Population } & \multicolumn{2}{|c|}{ Obesity events } & \multirow[t]{2}{*}{ p values } & \multicolumn{3}{|c|}{$\begin{array}{l}\text { Neighbourhood deprivation } \\
\text { rates of obesity }\end{array}$} & \multirow[t]{2}{*}{$\mathrm{p}$ values } \\
\hline & $\mathrm{n}$ & $\%$ & $\mathrm{n}$ & $\%$ & & low & moderate & high & \\
\hline Total population, $\%$ & 944,487 & & & & & $\begin{array}{l}212,529 \\
(22 \%)\end{array}$ & $\begin{array}{l}593,377 \\
(63 \%)\end{array}$ & $\begin{array}{l}138,581 \\
(15 \%)\end{array}$ & \\
\hline Total obesity events & & & 6,968 & & & 0.4 & 0.8 & 1.1 & \\
\hline Sex & & & & & $<0.001$ & & & & 0.011 \\
\hline Boys & 484,677 & 51.3 & 3,878 & 55.7 & & 0.4 & 0.8 & 1.2 & \\
\hline Girls & 459,810 & 48.7 & 3,090 & 44.3 & & 0.4 & 0.7 & 1.0 & \\
\hline Age, years & & & & & $<0.001$ & & & & $<0.001$ \\
\hline $0-4$ & 201,594 & 21.3 & 933 & 13.4 & & 0.2 & 0.5 & 0.7 & \\
\hline $5-9$ & 295,382 & 31.3 & 2,763 & 39.6 & & 0.5 & 1.0 & 1.4 & \\
\hline $10-14$ & 447,511 & 47.4 & 3,272 & 47.0 & & 0.4 & 0.7 & 1.2 & \\
\hline Family income & & & & & $<0.001$ & & & & 0.902 \\
\hline Low income & 236,965 & 25.0 & 1,918 & 27.5 & & 0.5 & 0.8 & 1.0 & \\
\hline Middle-low income & 236,067 & 25.0 & 1,626 & 23.4 & & 0.4 & 0.7 & 1.1 & \\
\hline Middle-high income & 235,762 & 25.0 & 1,889 & 27.1 & & 0.4 & 0.9 & 1.3 & \\
\hline High income & 235,693 & 25.0 & 1,535 & 22.0 & & 0.4 & 0.7 & 1.2 & \\
\hline Maternal marital status & & & & & $<0.001$ & & & & $<0.001$ \\
\hline Married/cohabiting & 554,811 & 58.7 & 3,684 & 52.9 & & 0.4 & 0.7 & 1.0 & \\
\hline $\begin{array}{l}\text { Never married, widowed, or } \\
\text { divorced }\end{array}$ & 389,676 & 41.3 & 3,284 & 47.1 & & 0.5 & 0.9 & 1.3 & \\
\hline Maternal educational attainment & & & & & $<0.001$ & & & & $<0.001$ \\
\hline$\leq 9$ years & 313,087 & 33.1 & 2,966 & 42.6 & & 0.6 & 1.0 & 1.2 & \\
\hline 10-11 years & 336,027 & 35.6 & 2,749 & 39.4 & & 0.5 & 0.8 & 1.2 & \\
\hline$\geq 12$ years & 295,373 & 31.3 & 1,253 & 18.0 & & 0.3 & 0.5 & 0.8 & \\
\hline Paternal educational attainment & & & & & $<0.001$ & & & & $<0.001$ \\
\hline$\leq 9$ years & 287,277 & 30.4 & 2,832 & 40.6 & & 0.6 & 1.0 & 1.2 & \\
\hline 10-11 years & 370,310 & 39.2 & 2,961 & 42.5 & & 0.5 & 0.8 & 1.2 & \\
\hline$\geq 12$ years & 286,900 & 30.4 & 1,175 & 16.9 & & 0.2 & 0.5 & 0.8 & \\
\hline Maternal immigrant status & & & & & $<0.001$ & & & & $<0.001$ \\
\hline Sweden & 795,272 & 84.2 & 5,544 & 79.6 & & 0.4 & 0.7 & 1.1 & \\
\hline Western countries & 57,246 & 6.1 & 481 & 6.9 & & 0.4 & 0.9 & 1.0 & \\
\hline Other countries & 91,969 & 9.7 & 943 & 13.5 & & 0.6 & 1.0 & 1.1 & \\
\hline Paternal immigrant status & & & & & $<0.001$ & & & & $<0.001$ \\
\hline Sweden & 795,510 & 84.2 & 5,501 & 78.9 & & 0.4 & 0.7 & 1.1 & \\
\hline Western countries & 65,251 & 6.9 & 550 & 7.9 & & 0.4 & 0.9 & 1.0 & \\
\hline Other countries & 83,726 & 8.9 & 917 & 13.2 & & 0.4 & 1.1 & 1.2 & \\
\hline Urban/rural status & & & & & 0.798 & & & & $<0.001$ \\
\hline Large cities & 238,476 & 25.2 & 1,866 & 26.8 & & 0.4 & 0.8 & 1.4 & \\
\hline Middle-sized towns & 471,316 & 49.9 & 3,279 & 47.1 & & 0.4 & 0.7 & 1.1 & \\
\hline Small towns/rural areas & 234,695 & 24.8 & 1,823 & 26.1 & & 0.5 & 0.8 & 0.9 & \\
\hline Move & & & & & 0.060 & & & & $<0.001$ \\
\hline Not moved & 639,552 & 67.7 & 4,644 & 66.6 & & 0.4 & 0.7 & 1.1 & \\
\hline Moved & 304,935 & 32.3 & 2,324 & 33.4 & & 0.4 & 0.9 & 1.1 & \\
\hline
\end{tabular}

Table 1 continued on next page

The results of our study are highly relevant in the understanding of the progress of the epidemic in overweight and obesity seen among school children world-wide as well as in the EU. The WHO has prioritized such data collection, and, today, 35 Member States of the WHO European Region participate in surveillance activities in order to monitor nutrition and prevent overweight and obesity in children [22]. These activities in the EU and the WHO high- 
Hamano et al.: Association between Childhood Obesity and Neighbourhood Accessibility to Fast-Food Outlets: A Nationwide 6-Year Follow-Up Study of 944,487 Children

Table 1. Continued

\begin{tabular}{|c|c|c|c|c|c|c|c|c|c|}
\hline & \multicolumn{2}{|c|}{ Population } & \multicolumn{2}{|c|}{ Obesity events } & \multirow[t]{2}{*}{$p$ values } & \multicolumn{3}{|c|}{$\begin{array}{l}\text { Neighbourhood deprivation } \\
\text { rates of obesity }\end{array}$} & \multirow[t]{2}{*}{$\mathrm{p}$ values } \\
\hline & $\mathrm{n}$ & $\%$ & $\mathrm{n}$ & $\%$ & & low & moderate & high & \\
\hline Maternal age at child birth, years & & & & & 0.045 & & & & $<0.001$ \\
\hline$<30$ & 418,029 & 44.3 & 3,202 & 46.0 & & 0.4 & 0.8 & 1.1 & \\
\hline $30-39$ & 493,643 & 52.3 & 3,499 & 50.2 & & 0.4 & 0.8 & 1.2 & \\
\hline$\geq 40$ & 32,815 & 3.4 & 267 & 3.8 & & 0.5 & 0.9 & 1.4 & \\
\hline Paternal age at child birth, years & & & & & 0.324 & & & & $<0.001$ \\
\hline$<30$ & 235,509 & 24.9 & 1,816 & 26.1 & & 0.4 & 0.8 & 1.2 & \\
\hline $30-39$ & 573,988 & 60.8 & 4,027 & 57.8 & & 0.4 & 0.8 & 1.1 & \\
\hline$\geq 40$ & 134,990 & 14.3 & 1,125 & 16.1 & & 0.5 & 0.9 & 1.3 & \\
\hline $\begin{array}{l}\text { Maternal hospitalisation of } \\
\text { diabetes }\end{array}$ & & & & & $<0.001$ & & & & $<0.001$ \\
\hline No & 937,540 & 99.3 & 6,803 & 97.6 & & 0.4 & 0.8 & 1.1 & \\
\hline Yes & 6,947 & 0.7 & 165 & 2.4 & & 1.3 & 2.4 & 3.0 & \\
\hline $\begin{array}{l}\text { Maternal hospitalisation of chronic } \\
\text { lower respiratory disease }\end{array}$ & & & & & $<0.001$ & & & & $<0.001$ \\
\hline No & 935,990 & 99.1 & 6,833 & 98.1 & & 0.4 & 0.8 & 1.1 & \\
\hline Yes & 8,497 & 0.9 & 135 & 1.9 & & 0.9 & 1.6 & 2.2 & \\
\hline $\begin{array}{l}\text { Maternal hospitalisation of alco- } \\
\text { holism and related liver disease }\end{array}$ & & & & & $<0.001$ & & & & $<0.001$ \\
\hline No & 937,255 & 99.2 & 6,877 & 98.7 & & 0.4 & 0.8 & 1.1 & \\
\hline Yes & 7,232 & 0.8 & 91 & 1.3 & & 0.7 & 1.3 & 1.5 & \\
\hline Paternal hospitalisation of diabetes & & & & & $<0.001$ & & & & $<0.001$ \\
\hline No & 931,554 & 98.6 & 6,746 & 96.8 & & 0.4 & 0.8 & 1.1 & \\
\hline Yes & 12,933 & 1.4 & 222 & 3.2 & & 0.7 & 1.6 & 2.4 & \\
\hline $\begin{array}{l}\text { Paternal hospitalisation of chronic } \\
\text { lower respiratory disease }\end{array}$ & & & & & $<0.001$ & & & & $<0.001$ \\
\hline No & 938,676 & 99.4 & 6,863 & 98.5 & & 0.4 & 0.8 & 1.1 & \\
\hline Yes & 5,811 & 0.6 & 105 & 1.5 & & 1.1 & 1.7 & 2.8 & \\
\hline $\begin{array}{l}\text { Paternal hospitalisation of alco- } \\
\text { holism and related liver disease }\end{array}$ & & & & & $<0.001$ & & & & $<0.001$ \\
\hline No & 928,168 & 98.3 & 6,775 & 97.2 & & 0.4 & 0.8 & 1.1 & \\
\hline Yes & 16,319 & 1.7 & 193 & 2.8 & & 0.9 & 1.1 & 1.3 & \\
\hline Hospitalisation of diabetes & & & & & $<0.001$ & & & & $<0.001$ \\
\hline No & 939,037 & 99.4 & 6,883 & 98.8 & & 0.4 & 0.8 & 1.1 & \\
\hline Yes & 5,450 & 0.6 & 85 & 1.2 & & 1.1 & 1.4 & 1.9 & \\
\hline Family history of obesity & & & & & $<0.001$ & & & & $<0.001$ \\
\hline No & 916,819 & 97.1 & 5,881 & 84.4 & & 0.4 & 0.7 & 1.0 & \\
\hline Yes & 27,668 & 2.9 & 1,087 & 15.6 & & 2.9 & 4.0 & 4.4 & \\
\hline
\end{tabular}

light the importance of collecting relevant data for policy developments in the area of combating the emerging obesity epidemic in the European Region.

Our results are consistent with recent research from the UK, where an association between the number of fast-food outlets per area unit and childhood obesity was found [9]. Another study conducted in 8- to 9-year-old Australian children, however, did not find similar associations [23]. One possible explanation for this discrepancy is the definition of neighbourhoods. The Australian study defined neighbourhood accessibility to fast-food outlets based on buffer zones from the children's home address. In the present study, neighbourhood accessibility defined by buffer zone also failed to find significant associations after adjustment for a comprehensive set of covariates (table 3). The accessibility defined by SAMS (table 2) 
Hamano et al.: Association between Childhood Obesity and Neighbourhood Accessibility to Fast-Food Outlets: A Nationwide 6-Year Follow-Up Study of 944,487 Children

Table 2. Association between childhood obesity and neighbourhood accessibility to fast food outlets, small area market unit ${ }^{\mathrm{a}}$

\begin{tabular}{|c|c|c|c|c|c|c|c|c|}
\hline & \multicolumn{2}{|c|}{ Model $1^{b}$} & \multicolumn{2}{|c|}{ Model $2^{c}$} & \multicolumn{2}{|c|}{ Model $3^{d}$} & \multicolumn{2}{|c|}{ Model 4e } \\
\hline & OR & $95 \% \mathrm{CI}$ & OR & $95 \% \mathrm{CI}$ & OR & $95 \% \mathrm{CI}$ & $\mathrm{OR}$ & $95 \% \mathrm{CI}$ \\
\hline \multicolumn{9}{|c|}{ Fast-food outlets } \\
\hline No accessibility & 1.00 & & 1.00 & & 1.00 & & 1.00 & \\
\hline Accessibility & 1.14 & $1.07-1.22$ & 1.07 & $1.00-1.14$ & 1.06 & $1.00-1.13$ & 1.06 & $1.00-1.13$ \\
\hline ICC & & 0.156 & & 0.133 & & 0.124 & & 0.104 \\
\hline
\end{tabular}

OR = Odds ratio; 95\% CI = 95\% confidence interval, ICC = intraclass correlation coefficient.

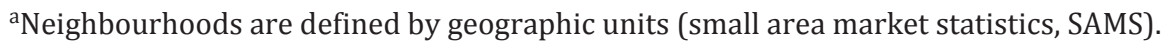

bModel 1 is unadjusted.

${ }^{c}$ Model 2 is adjusted for neighbourhood-level deprivation and individual-level age and sex.

${ }^{\mathrm{d}}$ Model 3 is adjusted for neighbourhood-level deprivation, individual-level age, sex, maternal marital status, family income, parental educational level, parental immigrant status, region of residence, mobility, and parental age at child birth.

${ }^{\mathrm{e}}$ Model 4: full model, model 3 + family history of obesity, and individual and parental hospitalisations.

Table 3. Association between childhood obesity and 'immediate' neighbourhood accessibility to fast food outlets, buffer zones ${ }^{\mathrm{a}}$

\begin{tabular}{|c|c|c|c|c|c|c|c|c|}
\hline & \multicolumn{2}{|c|}{ Model 1} & \multicolumn{2}{|c|}{ Model 2} & \multicolumn{2}{|c|}{ Model 3} & \multicolumn{2}{|c|}{ Model 4} \\
\hline & $\mathrm{OR}$ & $95 \% \mathrm{CI}$ & $\mathrm{OR}$ & $95 \%$ CI & $\mathrm{OR}$ & $95 \%$ CI & $\mathrm{OR}$ & $95 \%$ CI \\
\hline \multicolumn{9}{|c|}{ Fast-food outlets } \\
\hline No accessibility & 1.00 & & 1.00 & & 1.00 & & 1.00 & \\
\hline Accessibility & 1.07 & $1.01-1.14$ & 1.03 & $0.97-1.09$ & 0.99 & $0.94-1.05$ & 0.99 & $0.94-1.05$ \\
\hline ICC & & 0.157 & & 0.134 & & 0.125 & & 0.105 \\
\hline
\end{tabular}

OR = Odds ratio; $95 \% \mathrm{CI}=95 \%$ confidence interval, $\mathrm{ICC}=$ intraclass correlation coefficient.

${ }^{a}$ Neighbourhoods are defined by buffer zones (radius $1000 \mathrm{~m}$ ) surrounding each person.

bModel 1 is unadjusted.

${ }^{\mathrm{c}}$ Model 2 is adjusted for neighbourhood-level deprivation and individual-level age and sex.

${ }^{\mathrm{d}}$ Model 3 is adjusted for neighbourhood-level deprivation, individual-level age, sex, maternal marital status, family income, parental educational level, parental immigrant status, region of residence, mobility, and parental age at child birth.

eModel 4: full model, model 3 + family history of obesity, and individual and parental hospitalisations.

may more accurately reflect the neighbourhood as SAMS are defined by homogeneous types of buildings and have natural boundaries defined by highways or rivers, whereas buffer zones do not take such natural neighbourhood borders into account. Our results suggest that measuring exposure based on relatively homogeneous neighbourhoods with natural boundaries may be more representative to properly examine the associations between accessibility to fast-food outlets and individual-level obesity.

A likely explanation behind the association between neighbourhood accessibility to fastfood outlets and childhood obesity may be the unhealthy diets served on such outlets. Fast food is energy-dense and associated with poor food choices, such as low vegetable and milk intake $[8,24]$. 
Our study has certain limitations. Our results did not take into account individual-level dietary habits and physical activity patterns, which is impossible on an entire population. Although we included data from an entire country, further large-scale studies in other countries are needed to determine whether the findings can be generalised to other cultural settings. This highlights the importance of collecting data from several countries in large collaborative projects, such as in ongoing surveillance activities initiated by the WHO in European countries. It is also important to keep in mind that neighbourhoods that have fast-food outlets may also have other types of health-damaging environments (e.g. high crime rates, low walkability and few green areas), i.e. factors that we partly adjusted for in the present study that included the covariate neighbourhood deprivation. Future studies could explore the associations of fastfood outlets with other physical environmental characteristics in more detail.

The present study has also several strengths. First, the prospective design of our study is stronger than a cross-sectional design, suggesting a potential effect of the exposure. Second, this study analysed data on a large national cohort of children aged 0-14 years $(944,487$ children), and 6,968 cases with occurrences of obesity were identified during the study period, using data from both in- and out-patients. Third, SAMS units with an average of 1,000 residents were used to define a neighbourhood, which increases the probability that the children were actually exposed to the neighbourhood resources. Finally, the multilevel approach examined the neighbourhood effect on individual-level outcomes, i.e. childhood obesity, after adjustment for neighbourhood- and individual-level socio-demographic factors.

\section{Conclusions}

This prospective nationwide study showed that the neighbourhood accessibility to fastfood outlets was independently associated with increased odds of diagnosed childhood obesity. This finding implicates that residential environments should be considered when developing health promotion programmes.

\section{Acknowledgement}

This research was supported by grants from the Swedish Research Council and ALF Region Skåne, Sweden, to Kristina Sundquist and Jan Sundquist, and MEXT KAKENHI (grant number: 15K12317) to Tsuyoshi Hamano. Research reported in this publication was also supported by the National Heart, Lung, And Blood Institute of the National Institutes of Health under Award Number R01HL116381 to Kristina Sundquist. Funding sources were not involved in the study design, collection, analysis and interpretation of data, and in the preparation of the manuscript.

\section{Disclosure Statement}

The authors declare no conflict of interest.

\section{References}

1 Karnik S, Kanekar A: Childhood obesity: a global public health crisis. Int J Prev Med 2012;3:1-7.

2 Silventoinen K, Rokholm B, Kaprio J, Sørensen TI: The genetic and environmental influences on childhood obesity: a systematic review of twin and adoption studies. Int J Obes 2010;34:29-40.

3 Diez Roux AV, Mair C: Neighborhoods and health. Ann N Y Acad Sci 2010;1186:125-145.

4 Bammann K, Gwozdz W, Lanfer A, Barba G, De Henauw S, Eiben G, Fernandez-Alvira JM, Kovács E, Lissner L, Moreno LA, Tornaritis M, Veidebaum T, Pigeot I, IDEFICS Consortium: Socioeconomic factors and childhood overweight in Europe: results from the multi-centre IDEFICS study. Pediatr Obes 2013;8:1-12. 
Hamano et al.: Association between Childhood Obesity and Neighbourhood Accessibility to Fast-Food Outlets: A Nationwide 6-Year Follow-Up Study of 944,487 Children

5 Li X, Memarian E, Sundquist J, Zöller B, Sundquist K: Neighbourhood deprivation, individual-level familial and socio-demographic factors and diagnosed childhood obesity: a nationwide multilevel study from Sweden. Obes Facts 2014;7:253-263.

6 El-Sayed AM, Scarborough P, Galea S: Socioeconomic inequalities in childhood obesity in the United Kingdom: a systematic review of the literature. Obes Facts 2012;5:671-692.

7 Papas MA, Alberg AJ, Ewing R, Helzlsouer KJ, Gary TL, Klassen AC: The built environment and obesity. Epidemiol Rev 2007;29:129-143.

8 Bowman SA, Gortmaker SL, Ebbeling CB, Pereira MA, Ludwig DS: Effects of fast-food consumption on energy intake and diet quality among children in a national household survey. Pediatrics 2004;113:112-118.

9 Fraser LK, Edwards KL: The association between the geography of fast food outlets and childhood obesity rates in Leeds, UK. Health Place 2010;16:1124-1128.

10 Sturm R, Datar A: Body mass index in elementary school children, metropolitan area food prices and food outlet density. Public Health 2005;119:1059-1068.

11 Rosen M, Hakulinen T: Use of disease registers; in Ahrens W, Pigeot I (eds): Handbook of Epidemiology. Berlin, Springer, 2005, pp 232-251.

12 Teleadress: https://www.bisnode.com/sverige/tjanster/teleadress/(last accessed November 15, 2017).

13 Kawakami N, Winkleby M, Skog L, Szulkin R, Sundquist K: Differences in neighborhood accessibility to healthrelated resources: a nationwide comparison between deprived and affluent neighborhoods in Sweden. Health Place 2011;17:132-139.

14 Sundquist K, Malmstrom M, Johansson SE: Neighborhood deprivation and incidence of coronary heart disease: a multilevel study of 2.6 million women and men in Sweden. J Epidemiol Community Health 2004;58:71-77.

15 Sundquist K, Winkleby M, Ahlen H, Johansson SE: Neighborhood socioeconomic environment and incidence of coronary heart disease: a follow-up study of 25,319 women and men in Sweden. Am J Epidemiol 2004;159: 655-662.

16 Hamano T, Li X, Tanito M, Nabika T, Shiwaku K, Sundquist J, Sundquist K: Neighborhood deprivation and risk of age-related eye diseases: a follow-up study in Sweden. Ophthalmic Epidemiol 2015;22:308-320.

17 Sundquist J, Johansson SE, Yang M, Sundquist K: Low linking social capital as a predictor of coronary heart disease in Sweden: a cohort study of 2.8 million people. Soc Sci Med 2006;62:954-963.

18 Statistics Sweden. Localities 2010: Buildings and Urban Structures. 2012. ISSN 1654-3823.

19 James P, Berrigan D, Hart JE, Hipp JA, Hoehner CM, Kerr J, Major JM, Oka M, Laden F: Effects of buffer size and shape on associations between the built environment and energy balance. Health Place 2014;27:162-170.

20 Winkleby M, Sundquist K, Cubbin C: Inequities in CHD incidence and case fatality by neighborhood deprivation. Am J Prev Med 2007;32:97-106.

21 Snijders T, Bosker R: Multilevel Analysis: An Introduction to Basic and Advanced Multilevel Modeling. Thousand Oaks, Sage Publications, 1999.

22 World Health Organization Regional Office for Europe: WHO European Childhood Obesity Surveillance Initiative (COSI). www.euro.who.int/en/health-topics/disease-prevention/nutrition/activities/who-europeanchildhood-obesity-surveillance-initiative-cosi (last accessed November 15, 2017).

23 Crawford DA, Timperio AF, Salmon JA, Baur L, Giles-Corti B, Roberts RJ, Jackson ML, Andrianopoulos N, Ball K: Neighbourhood fast food outlets and obesity in children and adults: the CLAN Study. Int J Pediatr Obes 2008; 3:249-256.

24 Paeratakul S, Ferdinand DP, Champagne CM, Ryan DH, Bray GA: Fast-food consumption among US adults and children: dietary and nutrient intake profile. J Am Diet Assoc 2003;103:1332-1338. 